\title{
INTERNATIONAL FIELD INTERCOMPARISON OF ATMOSPHERIC MERCURY MEASUREMENT METHODS
}

\author{
W.H. SCHROEDER ${ }^{1}$, G. KEELER ${ }^{2}$, H. KOCK ${ }^{3}$, P. ROUSSEL ${ }^{4}$ \\ D. SCHNEEBERGER ${ }^{5,6}$, F. SCHAEDLICH ${ }^{6}$ \\ ${ }^{1}$ Environment Canada:AES, 4905 Dufferin Street, Downsview, Ontario M3H 5T4 CANADA. ${ }^{2}$ School of \\ Public Health, The University of Michigan, Ann Abor, Michigan 48109-2029, U.S.A. ${ }^{3}$ GKSS Reseanch \\ Centre, Chemistry Institute, P.O. Bor 1160, D-21494 Geesthacht, GERMANY. ${ }^{4}$ Ontario Hydro, Reseanch \\ Division, 800 Kipling Avenue, Tononto, Ontanio M8Z 5S4 CANADA. 5 Ontario Ministry of the Environment \\ and Energy, Resources Road, Etobicoke, Ontario M9P 3V6 CANADA. - Tekran Inc., 169 Oakwood Avenue, \\ Toronto, Ontario MGE 2VI CANADA
}

\begin{abstract}
To determine the extent of comparability of sampling and analytical procedures for atmospheric mercury $(\mathrm{Hg})$ being used by different scientific groups around the world and hence the compatibility of measurement results, the Atmospheric Environment Service (AES) co-ordinated a field intercomparison study in Windsor, Ontario, over a period of 5 days during Sept./Oct.,1993. This study brought together 2 groups (University of Michigan Air Quality Laboratory; Chemistry Institute of GKSS) which performed conventional (manual) sample collection procedures for total gaseous mercury (TGM) and for particulate-phase mercury (PPM), followed by cold-vapor atomic fluorescence spectrophotometric (CVAFS) analysis in the respective laboratories. Two other groups (Ontario Hydro, and the Ontario Ministry of Environment \& Energy) each operated a novel mercury vapor analyzer produced by Tekran Inc. of Toronto. As is the case for the manual methods, this analyzer also uses gold amalgamation and CVAFS. During the intercomparison, meteorological parameters (air temperature, barometric pressure, wind speed/direction and relative humidity) were obtained at the study site.
\end{abstract}

\section{Introduction}

Significant advances in the determination of $\mathrm{Hg}$ in various environmental media (at trace or ultra-trace levels) have occurred during the last 10 to 15 years (Fitzgerald and Gill, 1979; Schroeder,1982; Bloom and Fitzgerald,1988; Bloom,1989; Lindqvist,1991; Baeyens,1992). Atmospheric Hg measurements (this term includes both sampling and analytical operations) are being made with increasing frequency worldwide due to a growing awareness of the importance of the atmosphere in globally dispersing this heavy metal. In outdoor air--especially at rural or remote locations--Hg exists primarily in the vapor phase, with at most a few $\%$ of the total airborne $\mathrm{Hg}$ being associated with atmospheric aerosols. Moreover, almost all (generally > 95\%) of the vapor-phase $\mathrm{Hg}$ consists of the elemental form $\left(\mathrm{Hg}^{\circ}\right.$ atoms). In urban or industrial centers, the physical forms and/or chemical speciation of $\mathrm{Hg}$ in the atmosphere may, however, be significantly different.

Because of the extremely low concentrations at which $\mathrm{Hg}$ species normally exist in the atmosphere, current sampling techniques involve a pre-concentration step (filtration in the case of PPM; amalgamation with gold for vapor-phase $\mathrm{Hg}$ species). The latter air sampling technique (Schroeder et al.,1985) gives rise to the operational definition of "total gaseous $\mathbf{H g}$ ". Considerably less standardization of methods has occurred for the collection of PPM than for the collection of TGM. For PPM, air filtration is used almost exclusively, but the types of filter media employed vary considerably (e.g., quartz-wool plugs, glass fibre, Teflon or polycarbonate filter disks or organic/inorganic membranes). In most instances, after thermal release of the analyte in the form of $\mathrm{Hg}^{\circ}$, either from the gold collector via "de-amalgamation" or from the filtered particulate matter via pyrolysis, detection and quantitation is achieved by CVAFS. Conventional (nanual) methods for atmospheric $\mathrm{Hg}$ measurements employ a sampling train consisting of a filter medium to retain PPM folllowed by an adsorbent-béd (e.g.,activated charcoal or proprietary products such as Carbosieve ${ }^{\mathrm{R}}$, Carbotrap ${ }^{\mathrm{R}}$, or Tenax ${ }^{\mathrm{R}}$ ) or a noble metal "trap" (usually $\mathrm{Au}$ or $\mathrm{Ag}$ ) which amalgamates gaseous $\mathrm{Hg}$ species (Dumarey et al., 1985; Schroeder and Jackson,1985; Schroeder et al.,1985). 
Given the remarkable resurgence of interest and the impressive level of effort expended in recent years on atmospheric $\mathrm{Hg}$ measurements in North America and Europe, it is very important to intercompare the sampling and analytical methods used by different groups of investigators. Collaborative methodology intercomparisons are an effective way of testing the "maturity" and "ruggedness" of sampling and analytical protocols (ACS,1980; ASTM,1977; Youden and Steiner,1975). So far, standardization of methods and procedures for atmospheric $\mathrm{Hg}$ measurements has occurred only to a rather limited extent. Yet, our understanding of the atmospheric chemistry and the complex environmental behavior of $\mathrm{Hg}$ species released into the troposphere from a diverse array of anthropogenic and natural sources is critically dependent upon accurate and/or precise measurement methods. The recent development (by Tekran Inc. of Toronto) of an instrument which automates the sampling and analytical steps in atmospheric $\mathrm{Hg}$ measurements also provided an impetus for a field intercomparison between traditional methods of sampling and analysis and the $\mathrm{Hg}$ vapor analyzer described in this paper. In an effort to determine the intercomparability of sampling and analytical procedures currently in use and the degree of compatibility of measurement results obtained with environmental samples, AES co-ordinated a field intercomparison study in Windsor, Ont., over a period of 5 days during September/October,1993. To our knowledge,this is the first multi-national field intercomparison of atmospheric $\mathrm{Hg}$ measurement methodologies undertaken in North America (or perhaps anywhere).

\section{Experimental Description}

This intercomparison took place from Monday, Sept. 27 to Friday, Oct.1, 1993, on the premises of the West Windsor Water Pollution Control Plant in the City of Windsor which is located in south-western Ontario, Canada. It involved 2 laboratories practicing conventional, manual sampling and analytical procedures for atmospheric $\mathrm{Hg}$ (both total gaseous $\mathrm{Hg}$ and particulate-phase $\mathrm{Hg}$ ) and 2 laboratories, each operating a Tekran $\mathrm{Hg}$ vapor analyzer. Project personnel and equipment were accommodated in an AES mobile laboratory parked on the study site. Manual sample collection methods followed an identical protocol: concurrent 6-hour and two 3-hour (or-three 2-hour) runs during the day-time, followed by an over-night run lasting about 15 to 18 hours. Results from the Tekran analyzers (obtained at 5-minute intervals) were averaged over the same time periods as for the manual methods. A total of 13 runs were completed: Duplicate samples for TGM and for PPM were taken during most runs.

\subsection{SAMPLING PROCEDURES}

A generic schematic of the $\mathrm{Hg}$ sampling trains used in the manual methods is given in Figure 1. Sampling flow rates were in the range of 300 to $500 \mathrm{~mL} / \mathrm{min}$.

Mercury associated with airborne particulate matter was retained on carefully pre-cleaned filter media. Lab A used glass-fiber filters (Gelman type A/E) in Teflon filter cassettes, whereas Lab B used traps consisting of quartz-wool plugs contained in quartz tubes $(6 \mathrm{~mm}$ o.d.; $4 \mathrm{~mm}$ i.d.). Filter media were changed on a daily basis. For determining PPM, Lab A collected separate samples on glass-fiber filters at a nominal flow rate of $30 \mathrm{~L} / \mathrm{min}$, whereas Lab B used the quartz-wool traps located ahead of the TGM sampling train.

For the collection of TGM, traps similar in design to that shown in Figure 2 were used. Lab A utilized traps ( $\sim 12 \mathrm{~cm}$ long) containing either Au-coated glass beads or Au-coated quartz sand, whereas Lab B used only Au-coated glass bead traps ( $7.5 \mathrm{~cm}$ long). Each lab employed 2 traps in series to check on collection efficiency. The collection efficiency of both types of traps has been found to be $\geq 95 \%$ (Dumarey et al,,1985).

A flow diagram for the Tekran Model 2537A Hg vapor analyzer is provided in Figure 3. TGM is collected onto gold. PPM is removed by a $47 \mathrm{~mm}$ diam. Teflon filter located immediately upstream of the Au cartridges. The analyzer was programmed to sample ambient air at a flow rate of $2 \mathrm{~L} / \mathrm{min}$ for 5 -minute sampling intervals. 

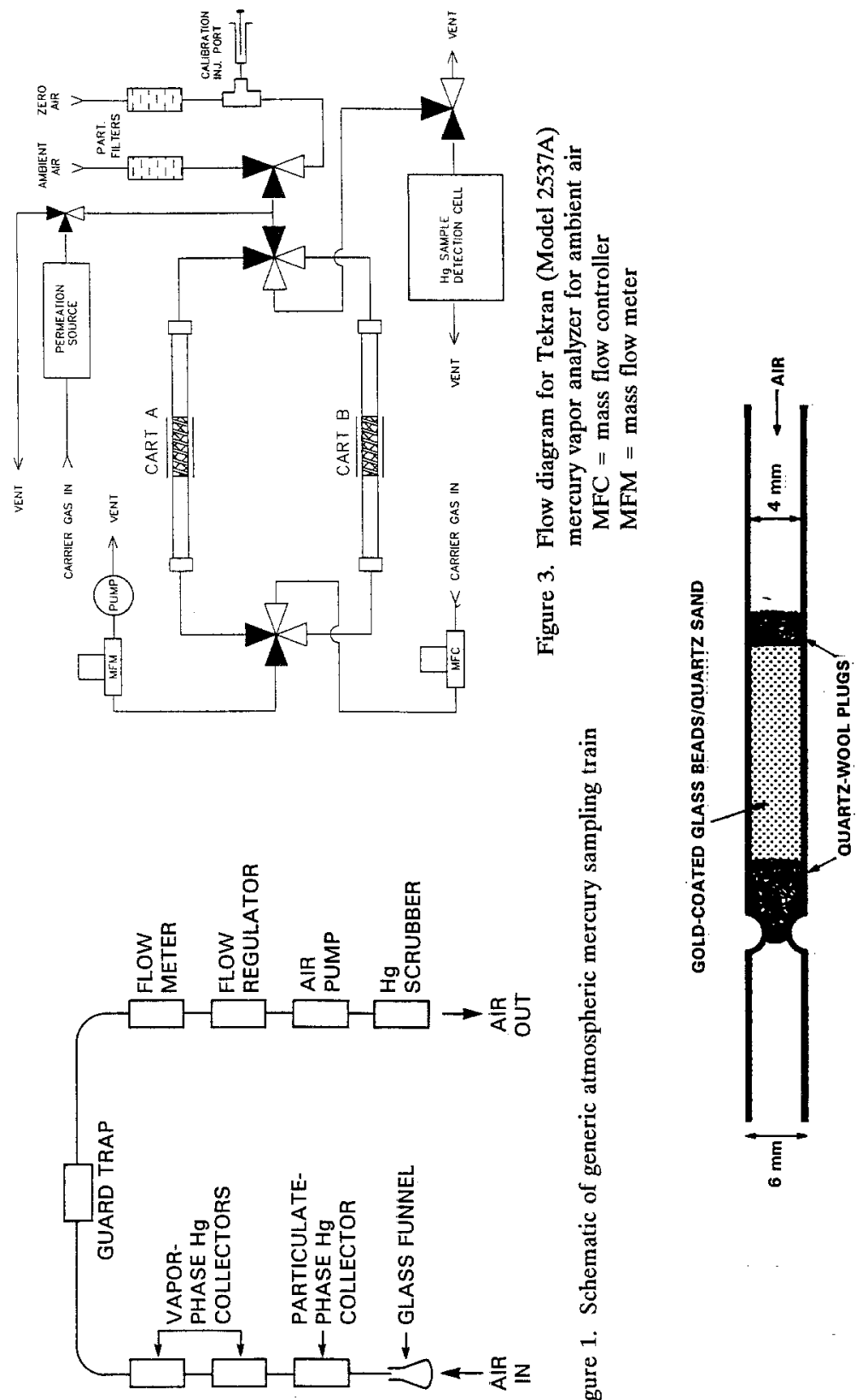

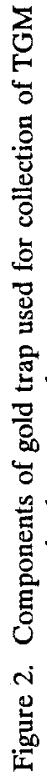


Meteorological parameters (air temperature, barometric pressure, wind speed/direction \& relative humidity) were measured at the study site with an instrumented $10 \mathrm{~m}$ tower located in an open field $\sim 150 \mathrm{~m}$ from the mobile lab containing the sampling and analytical apparatus. The met. data ( $5 \mathrm{~min}$ averages) were obtained with the following instrumentation: pressure = SETRA (SBP 270); temp.\& r.h. $=$ ROTRONIC Model MP-100F; temp. gradient $=2$ thermocouples with radiation shields at 1 \& $9 \mathrm{~m}$ above ground; wind speed \& direction = YOUNG Model 05103 anemometer. All parameters were collected with a $100 \%$ data capture rate over the entire study period.

\subsection{ANALYTICAL METHODOLOGY}

Figure 4 is a flow diagram describing the main components of the analytical systems employed for $\mathrm{Hg}$ analysis by the 2 labs using manual methods. The dual $\mathrm{Au}$ amalgamation/de-amalgamation technique (Fitzgerald and Gill,1979) was used in analyzing all samples generated by the manual methods:-In each instance, after thermal release of the analyte as $\mathrm{Hg}^{\circ}$, either from the TGM trap or from the PPM samples, detection and quantitation was achieved by CVAFS. Chemical analyses were performed in each lab by experienced personnel.

Each of the participating labs calibrated their CVAFS detector using a saturated $\mathrm{Hg}^{\circ}$ vapor calibration method essentially the same as that described in a 1987 Canadian Standards Assoc. report (CSA,1987). The Tekran analyzers contain a $\mathrm{Hg}^{\circ}$ permeation tube source (secondary standard) which was calibrated against manual injections from a saturated $\mathrm{Hg}^{\circ}$ vapor calibration unit. Both instruments were calibrated daily, using the perm. source calibration procedure: each cartridge in the instrument goes through a "clean", "zero", "span", and "clean" sequence of operations.

As is customary in environmental measurements of trace contaminants, each study participant used "field blanks" or "procedural blanks" which were processed through the entire series of sampling and analytical steps as if they were actual samples, except that they did not have ambient air pulled through them. The resulting "blank" values were subtracted from the analytical results ("raw data") obtained for the field samples. For the instrumental method, the procedural blank values were obtained by sampling (UHP grade) zero air ( $10 \mathrm{~L}$ ) through each of the two cartridges for the duration of one sampling period ( 5 minutes) before desorbing the cartridge. It should be noted, however, that this procedure is not directly comparable to a manual method field blank, since even UHP grade compressed gases can contain residual amounts of impurities, including $\mathrm{Hg}^{\circ}$ vapor. The procedural blank obtained this way would include the full contribution from any $\mathrm{Hg}$ contained in the zero gas and a true "field blank" for the instrumental method would be expected to be smaller than the values reported here. A summary of field and procedural blank values from the Windsor intercomparison study follows (Table I).

\section{Results and Discussion}

\subsection{TGM IN AIR}

The results obtained in the study for [TGM] in ambient air are shown in Figure 5. Of the 13 runs comprising this study, 8 produced meaningful results. For these 8 runs, all measurement results were in reasonably good agreement. The maximum deviation of individual results from the arithmetic mean of all 8 sets of results was about $35 \%$. Considering the difficulties inherent in making ultra-trace $\mathrm{Hg}$ measurements, this degree of agreement is quite satisfactory. For 4 runs $(\# 8,10,12,13)$ the agreement was especially good. Often, the mean value of the results produced by the Au-coated beads and the Aucoated sand traps used by Lab A was in satisfactory agreement with those from the Au-coated bead traps used by Lab B. Most of the time, the 2 Tekran instruments gave values that were somewhat lower than those produced by the manual methods. However, whereas there was considerable variability among the individual results reported by Labs $\mathrm{A}$ and $\mathrm{B}$, the 2 automated analyzers produced results which were consistently in very good agreement (see Figure 6). 


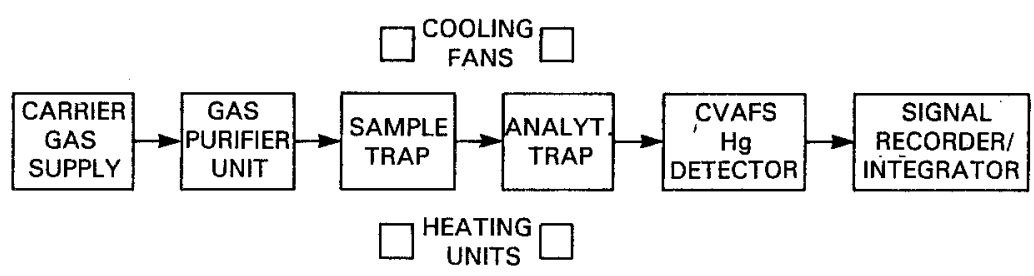

Figure 4. Schematic of generic atmospheric mercury analysis system

TABLE I

Summary of field blanks (manual methods) and zero air procedural blanks (automated instruments)

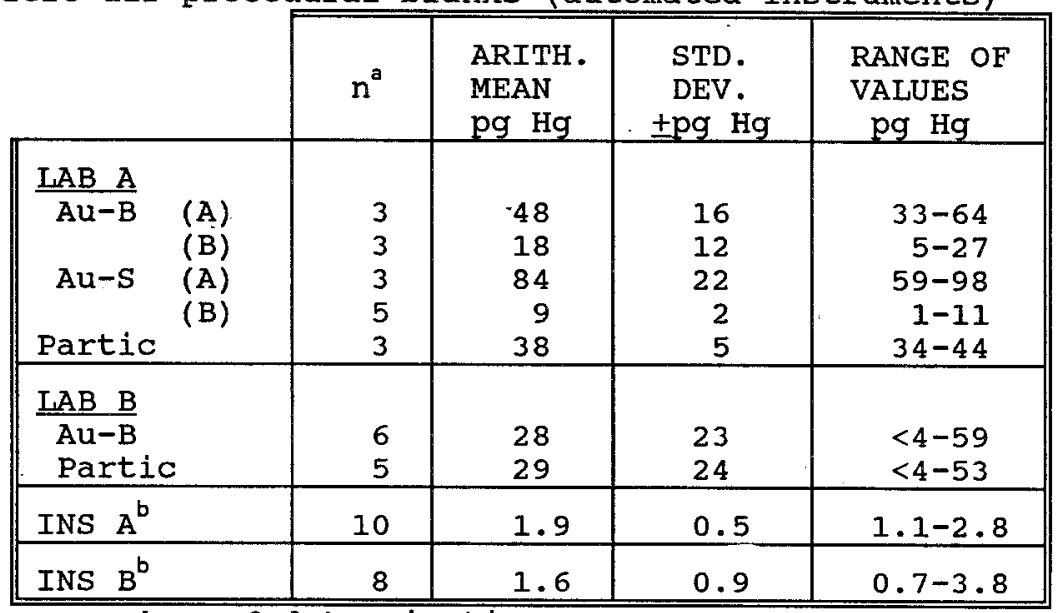

a: number of determinations

$\mathrm{b}$ : based on runs with UHP "zero air"

Au-B: traps containing gold-coated glass beads Au-S: traps containing gold-coated quartz sand

(A): for runs \#1-6

(B): for runs \#7-13

Partic: particulate-phase mercury filter media INS: TEKRAN Model 2537A automated Hg vapor analyzer 


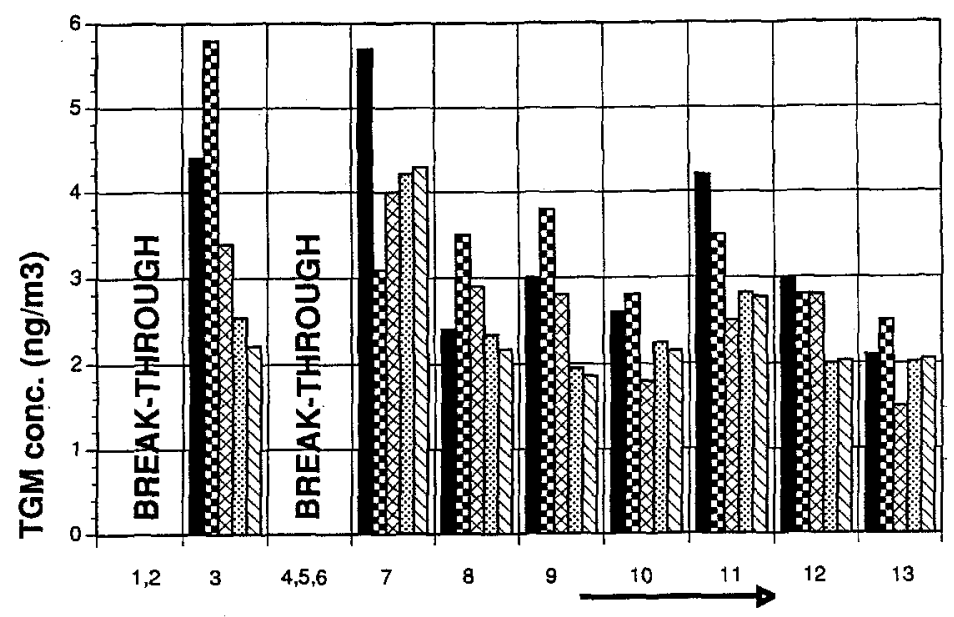

Run No.

\section{LAB A (Bead)}

\section{LAB A (Sand) 8888 INS A \% \\ LAB B (Bead) INS B WIIIV}

Figure 5. Total gaseous mercury (TGM) concentrations in $\mathrm{ng} / \mathrm{m}^{3}$ of air as reported by the 4 laboratories participating in the 1993 Windsor, Ontario, field intercomparison

\section{September 27 - October 1, 1993}

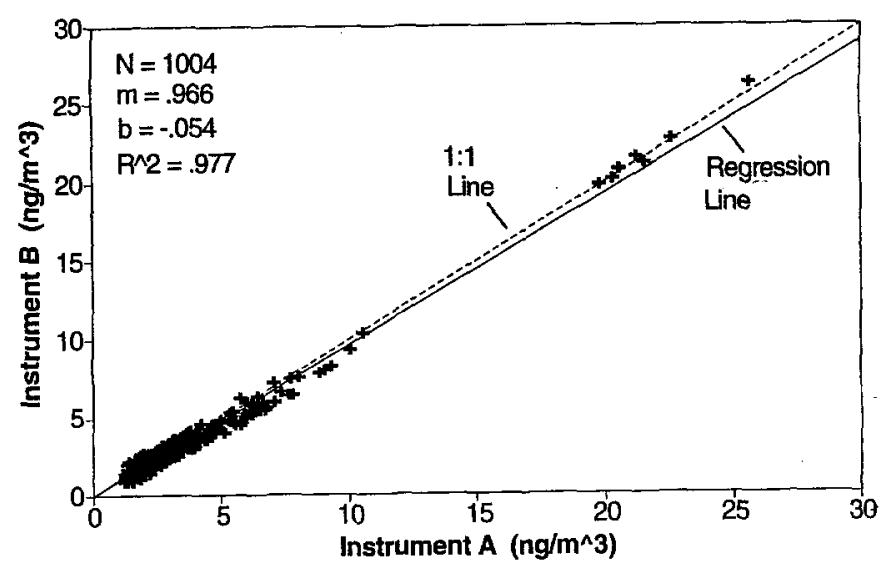

Figure 6. Correlation plot of TGM concentrations (ng/ $\mathrm{m}^{3}$ ) obtained with the 2 Tekran mercury vapor analyzers 
As identified in Figure 5, five of the first 6 runs of the manual methods displayed break-through of $\mathrm{Hg}$ from the 1st trap onto the 2nd. Break-through is deemed to have occurred when more than the usual quantity of $\mathrm{Hg}$ is found on the 2nd (back-up) Au trap. Normally the amount of mercury detected on the 2 nd trap is only a few \% of that found on the first trap. Without implementing special diagnostic tests, the automated analyzers would not detect the occurrence of break-through since they do not use $\mathrm{Au}$ cartridges in series. Such tests were carried out on 2 occasions later in the week, after preliminary results of chemical analyses of traps from some manual runs indicated the occurrence of break-through. In these tests, a known amount of $\mathrm{Hg}^{\circ}$ vapor was added to the ambient air stream near the end of a 5 -min sampling period to verify that the collection efficiency of the Tekran An cartridge had not been compromised during the sampling cycle. The contribution of $\mathrm{Hg}$ from ambient air was known from the other instrument. In both instances complete recovery of the spike was found, confirming that at least for those runs (\# 12 and 13) there was sufficient capacity for amalgamation of the $\mathrm{Hg}$ contained in the sample and in the spike.

For the 5 runs in which break-through occurred, the results obtained by Labs A and B were much higher than those obtained by the automated analyzers. Break-through of $\mathrm{Hg}$ on Au traps is a sampling artifact caused by "passivation" (i.e., de-activation) of the gold surface which collects the Hg contained in the air sample. Because both of the Au-coated sampling media utilized in the manual methods experienced considerable passivation (evidenced by break-through) the $\mathrm{Hg}$ vapor analyzers, since they too rely on the gold-amalgamation principle, may also have been affected. This could explain why the instruments gave lower values than the manual methods for runs 1,2,4,5, and 6. It cannot be ruled out, however, that for these runs the manual methods were subject to a positive bias (analytical interference) caused by one or more, as yet unknown, interfering substance(s). Sewage treatment plants may represent an especially challenging environment for atmospheric mercury measurements (Soldano et al.,1975).

During the runs which gave inconsistent results, the smell of hydrogen sulfide ("sewer gas") was quite strong. We know that $\mathrm{H}_{2} \mathrm{~S}$ and other 'reduced sulfur gases' (e.g., carbon disulfide, dimethyl sulfide, other alkyl sulfides \& di-sulfides, as well as mercaptans) are sorbed onto gold surfaces. Also, for most of the time during the "anomolous runs", it was raining at the study site. The following trend in meteorological conditions prevailed: lower barometric pressure ( $\sim 980$ to $\sim 990 \mathrm{mB}$ ); higher relative humidity (between 70 and $100 \%$ ); weaker inversion conditions; milder air temperatures $\left(\sim 8\right.$ to $\sim 14^{\circ} \mathrm{C}$ ), as compared to the rest of the week; wind direction between $\sim 200 \mathrm{deg}$ (southerly air flow) and $\sim 280 \mathrm{deg}$ (westerly air flow). One or more of these parameters may have contributed to the observed anomolies.

Determination of atmospheric $\mathrm{Hg}$ based on the Au-amalgamation sampling technique coupled with CVAFS detection is subject to (at least) 2 possible sources of interference: (a) chemical species (other than $\mathrm{Hg}$ ) present in air might interact with, be sorbed to, or be deposited on the gold surface (or the quartz-wool plugs in the traps used in the manual methods, but not the automated analyzers) so as to affect normal collection of $\mathrm{Hg}$ species. Indeed, one of the laboratories reported a distinct "sewer gas" odor during analyses of some traps used during the first 2 days of the study, clearly demonstrating that a significant quantity of sulfur compounds were collected along with (instead of ?) mercury. Potential chemical interferents include: hydrogen sulfide and other reduced sulfur compounds, gaseous ammonia, acidic gases such as $\mathrm{HCl}, \mathrm{HNO}_{3}, \mathrm{H}_{2} \mathrm{SO}_{4}, \mathrm{NO}_{2}, \mathrm{SO}_{2}, \mathrm{Cl}_{2}$ and $\mathrm{Br}_{2}$ (Cotten and Wilkinson,1962; McNerney et al.,1972; Ohkawa et al.,1976); (b) absorption of UV radiation (within the range of frequencies emitted by the instrument's light source) and fluorescence (or phosphorescence/light scattering/quenching) at the analytical wavelength by a substance in ambient air which is also retained on $\mathrm{Au}$ surfaces (initially in the sample trap and subsequently in the analytical trap) and then released into the detector cell along with the $\mathrm{Hg}^{\circ}$ vapor. Because of the multiple criteria which must be satisfied in case b), the occurrence of such an analytical interference is unlikely in the dual-stage amalgamation procedure for atmospheric $\mathrm{Hg}$ measurements. In practice, interferences of either type are not very common, at least in locations where the air is relatively clean/unpolluted.

Nevertheless, this is not the first time that measurement artifacts (methodological interferences) have been reported in connection with the use of gold (or other noble metals) as a collection medium for atmospheric Hg species (Schroeder et al.,1985). Thus, Slemr et al. (1979) reported that Au-coated quartz 
wool collectors are "de-activated" by flushing them with $500 \mathrm{~L}$ of urban or "maritime air" (air passed through sea-water). While this de-activation appeared to have no effect on the subsequent collection of $\mathrm{Hg}^{\circ}$, it did decrease the collection efficiency for dimethyl $\mathrm{Hg}$ from 94 to $73 \%$. In an extensive study performed in a rural setting on the west coast of Sweden, Brosset and Iverfeldt (1989) sometimes observed a reduction in the $\mathrm{Hg}$-amalgamating capacity of gold, increasing with the volume of air sampled through Au traps. This "blocking effect" resulted in lowered air concentration values for TGM at total sample volumes in excess of about $400 \mathrm{~L}$. They observed that the extent of this "blocking effect" seemed to depend on climatological parameters (but did not elaborate on this statement). Brosset and Iverfeldt also observed that the $\mathrm{Hg}$ which broke through the first trap was "in no case" collected by the second Au trap in their sampling train. They report that "It looks as if Au, successively and in all the traps almost simultaneously, gets its surface blocked towards amalgamation during the exposure." In our study at the Windsor WPCP, the second traps (in those cases for which break-through occurred) did contain much higher amounts of mercury than was found for the "normal" runs. This suggests that the de-activation phenomenon in these 2 cases may have involved different substances and/or different mechanisms of action. Brosset and Iverfeldt showed that the blocking layer contained volitile, watersoluble constituents, "which may indicate that it consists of $\mathrm{NH}_{4}$-salts, $\mathrm{NH}_{3}$ complexes, or other substances." They note that the extent of de-activation/deposition seemed to vary from case to case.

The presence of a measurement artifact during the early part (only) of our study is also indicated by a comparison of the results from sequential 2- or 3-hour sampling periods vs concurrent 6-hour runs (Table II). The runs in which the Au-traps were changed after 6 hours (volume-weighted average concentration) generally resulted in values that were significantly lower than the time-weighted average concentration derived from successive concurrent 2- or 3-h sampling periods. In the absence of any measurement artifacts, the results from the short-term and longer-term sampling periods should, of course, agree (within the total measurement error).

\subsection{PPM IN AIR}

We cannot say at this time, whether the conditions which led to break-through of gas-phase $\mathrm{Hg}$ from the first Au traps onto the back-up traps of the manual sampling trains, on the first two days of our methods intercomparison, also affected the results determined for particulate-phase $\mathrm{Hg}$ by Labs $\mathrm{A}$ and B. The PPM concentrations reported by the two labs practicing the manual method are given in Table III. Lab A (which used glass fiber filters at a sampling flow rate of $30 \mathrm{~L} / \mathrm{min}$ ) consistently obtained results that were much lower than those obtained by Lab B (which used quartz-wool plugs at a flow rate of 300 to $500 \mathrm{~mL} / \mathrm{min}$ ). Only for the second sample were the results of the 2 labs even close; for the first sample they differ by as much as a factor of 10 .

On the basis of some previous indications that small amounts (relative to $\mathrm{Hg}^{\circ}$ concentrations normally encountered in the atmosphere, but of a similar order of magnitude as PPM atmospheric loadings) of elemental $\mathrm{Hg}$ vapor could be retained on quartz-wool plugs, Lab B used the following analytical procedure. Prior to the particulate-phase $\mathrm{Hg}$ determinations, the quartz-wool traps (while being maintained at a temperature of $100^{\circ} \mathrm{C}$ ) were thoroughly flushed with argon gas for 4 minutes. However, for the PPM samples from Windsor, no significant release of $\mathrm{Hg}$ (taken here as $>10 \mathrm{pg}$ ) was observed with this pre-treatment.

\section{Summary and Conclusions}

During the autumn of 1993, scientists from Canada, the U.S.A. and Germany met in Windsor, Ontario to take part in a field intercomparison of vapor- and particulate-phase atmospheric $\mathrm{Hg}$ measurement methods. This sampling and analytical methods intercomparison involved manual ("traditional") procedures as well as a new automated $\mathrm{Hg}$ vapor analyzer. It provided a valuable opportunity for 
TABLE II

Comparison of time-wejghted average (successive 2- or 3 -h runs) and volumeweighted average TGM concentrations for concurrent 6-hour samples (as determined by the two laboratories using manual methods)

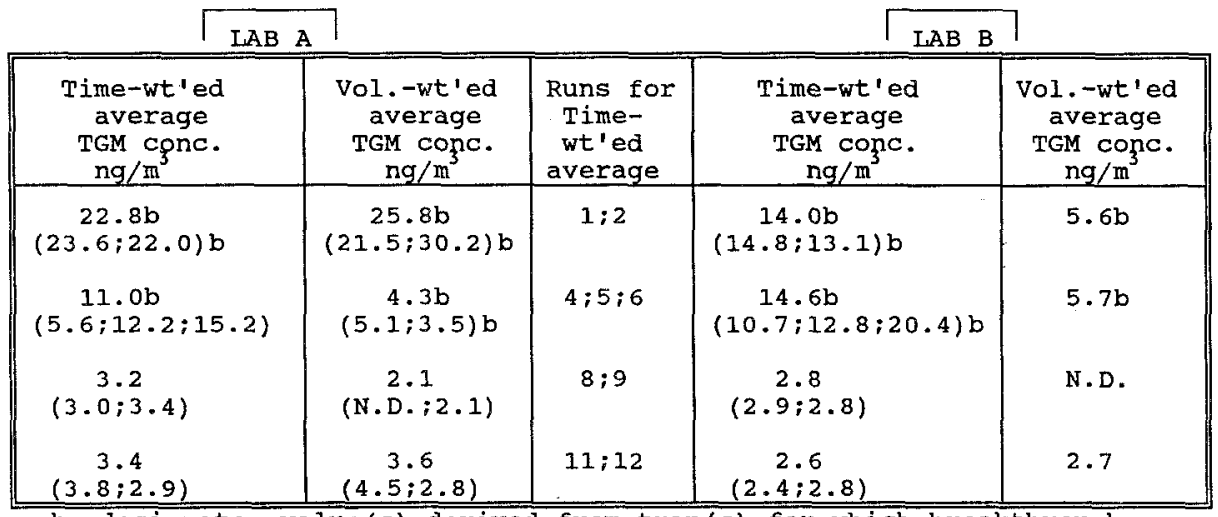

b: designates value(s) derived from trap(s) for which breakthrough occurred (i.e. higher than normal amount of $\mathrm{Hg}$ was found on the second (back-up) trap).

N.D.: no data. LAB A values in brackets, for "vol.-wt'ed average", are based on bead and sand traps, respectively.

TABLE III

Comparison of particulate-phase mercury concentrations $(24-\mathrm{h}$ average values) determined by Lab $A$ and Lab B

\begin{tabular}{|c|rr|ll|}
\hline $\begin{array}{c}\text { Sample } \\
\#\end{array}$ & \multicolumn{2}{|c|}{$\begin{array}{l}\text { Lab } \\
\mathrm{pg} / \mathrm{m}^{3}\end{array}$} & \multicolumn{2}{|c|}{$\begin{array}{l}\mathrm{Lab} \mathrm{m}^{3} \mathrm{~B} \\
\mathrm{pg} / \mathrm{m}^{3}\end{array}$} \\
\hline 1 & 82 & 81 & 810 & 600 \\
2 & 190 & 184 & 250 & 270 \\
3 & 107 & 102 & 2 peaks & 2 peaks \\
4 & 43 & 45 & 240 & 230 \\
\hline
\end{tabular}


collaborative testing of protocols for atmospheric $\mathrm{Hg}$ measurements under a range of field/meteorological conditions and methodological parameters. 13 runs were completed over a period of 5 days at the study site (a wastewater treatment plant). For 8 of the 13 runs, reasonable agreement on existing atmospheric TGM levels was obtained among all participants. However, in 5 of the 6 runs completed during the first 2 days of the study, measurement artifacts and inconsistent results were observed at this site (break-through of $\mathrm{Hg}$ from the 1st to the 2nd Au traps and unsatisfactory agreement between the manual and automated methods, and even among the two manual methods themselves). Agreement of results between the two labs which performed measurements of particulatephase $\mathrm{Hg}$ is deemed to be unsatisfactory.

Even though great strides in atmospheric $\mathrm{Hg}$ measurements have been made in recent years, making reliable determinations of gaseous and/or particulate-phase $\mathrm{Hg}$ species in ambient air is not a trivial task. The scientific community should not become too complacent and assume that all challenges in this area have already been met. Measurement methods which are known to perform reliably and behave consistently in relatively clean air masses, as are generally encountered in rural locations or at remote sites, may not display the same levels of reliability and consistency in urban environments or industrial settings. Mercury is a multi-faceted chemical element which continues to display new or unexpected behavior and resists attempts to easily/readily determine the presence and abundance in the atmosphere of its ecologically/toxicologically significant species. Any given methodology for sampling and analysis of $\mathrm{Hg}$ present in the atmosphere (or other environmental media) must always be validated carefully in any new or unusual surroundings/circumstances.

\section{Acknowledgments}

We would like to thank the following people without whom this study would not have succeeded: T. Dann, Environment Canada(financial support); W.Windsor WPCP technical/management personnel; A. Ng, OMEE Mobile Monitoring Centre; A. Tham, AES (technical support); J. Burke, T. Dvonch, M. Hoyer, A. Rea, and A. Vette of UMAQL (sampling and analyses); J. Metcalfe, AES (meteorological equipment loan); D. Yurkiewich (AES) and C. Schroeder (word processing).

\section{References}

ACS Committee on Environmental Improvement: 1980, Anal. Chem. 52, 2242-2249.

ASTM: 1977, Standards on Precision \& Accuracy for Various Applications. American Society for Testing and Materials, Phil.,PA. Baeyens, W.: 1992, Trends Anal. Chem. 11: 245-254.

Bloom, N.S.: 1989, Can. J. Fish. Aquat. Sci. 46, 1131-1140.

Bloom, N.S. and Fitzgerald, W.F.: 1988, Anal. Chim. Acta 208, 151-161.

Brosset, C. and Iverfeldt, A.: 1989, Water, Air, Soil Pollut. 43, 147-168.

CSA: 1987, Measurement of total mercury in air - cold vapour atomic absorption spectrophotometric method. Report

CAN/CSA-Z223.26-M87, Canadian Standards Association, Rexdale (Toronto), Ontario, Canada.

Cotton, F.A. and Wilkinson, G.: 1962, Advanced Inorganic Chemistry, Interscience Publishers, London, U.K.

Dumarey, R, Dams, R. and Hoste, J.: 1985, Anal. Chem. 57, 2638-2643.

Fitzgerald, W.F. and Gill, G.A.: 1979, Anal. Chem. 51, 1714-1720.

Lindqvist, O. (Ed.): 1991, Water, Air, Soil Pollut. 55(Sp.Ed.) Ch.3; App.I

McNerney, J.J., Buseck, P.R. and Hanson, R.C.: 1972, Science 178, 611-612.

Ohkawa, T., Uenoyama, H. and Kondo, M.: 1976, Eisei Kagaku [J. Hygienic Chemistry] 22, 11-19.

Schroeder, W.H.: 1982, Environ. Sci. Technol. 16, 362A-400A.

Schroeder, W.H. and Jackson, R.A.: 1985, Intern. J. Environ. Anal. Chem. 22, 1-18.

Schroeder, W.H., Hamilton, M.C. and Stobart, S.R.: 1985, Revs. Anat. Chem. \&, 179-209.

Slemr, F., Seiler, W., Eberling, C. and Roggendorf, P.: 1979, Anal. Chim. Acta 110, 35-47.

Soldano, B.A., Bien, P. and Kwan, P.: 1975, Atmos. Environ. 9, 941-944; 10, 171-172.

Youden, W.J. and Steiner, E.H.: 1975, Statistical Manual Association of Official Analytical Chemists. AOAC, Arlington, VA. 